\title{
A New Digital Video Watermarking Algorithm
}

\author{
Ren Xiang-jun 1,2, a , Wei Zhi-qiang ${ }^{1, b}$, Liang Zuo-juan ${ }^{1, c}$, Zhang Wei-jie ${ }^{3, d}$,
Dong Yun-gang \\ ${ }^{1}$ College of Information Science and Engineering, Ocean University of China, Qingdao, Shandong, \\ China \\ ${ }^{2}$ Qingdao TV Station, Qingdao, Shandong, China \\ ${ }^{3}$ Haier White Goods Group, Qingdao, Shandong, China \\ ${ }^{4}$ China Unicom Weifang Branch, Weifang, Shandong, China \\ arxj_1110@126.com, 'beizhiqiang@ouc.edu.cn, Izuojuan@ouc.edu.cn, 'zhangwj@haier.com, \\ eDongyungang@gmail.com
}

Keywords: video watermarking; MPEG-4 streams; information compression

\begin{abstract}
A new digital video watermarking algorithm for TV Program stream was proposed. Through technologies of thinning image and skeleton scaling, the size of embedded data is reduced. The compressed watermark signal is embedded into the unchanged area of I frame. The experimental results demonstrate that this method can not only ensure the desirable invisibility, but also avoid the computing cost of decoding.
\end{abstract}

\section{Introduction}

Digital media has already been widely used in public recreation now. Due to fast spread speed and low cost for reproduction, as a main media, digital video will cause some problems. For example, it is more easier to infringe the copyright and to temper with the works. Digital watermarking technology can carry copyright protection information. Its initial study focuses on image watermarking and it already has many research results. But the research based on digital video is still relatively small, so it is a very active field of watermarking research currently.

\section{Related Work}

Digital video watermarking research is basically carried out simultaneously with the image watermarking. According to video encoding and embedding strategy, we can divide video watermarking into three kinds of methods which include embedding into the uncompressed original video image, video codec and the compressed video streams.

For the first kind of method, on the basis of image watermarking, video is decomposed into two-dimensional image sequences. After adding watermarking into each frame, it needs to be re-compressed. This method does not take advantage of the correlation of the front and rear frames. Deguillaume treated the time axis of video as the third dimension of the two-dimensional image and embedded spread spectrum watermark into the 3D-DFT coefficient in video sequence[1]. Zhang Li-he embedded watermark information into the amplitude of video signal 3D Gabor transform coefficient. They make full use of the time dimension of video, but the computational complexity is great and the computation cost of decoding is relatively heavy. So it is not suitable for digital TV[2].

The second kind of method is video coedc method. The watermark is embedded into the coefficients of the transform domain during the encoding phase. The advantage of this method is that it will not increase the video data bit rate and its disadvantage is it will reduce the video quality[3].

The third kind of method is to embed watermark into the compressed video streams. Watermark is directly embedded into the compressed bit streams. The significant advantage of this method is it does not have the process of decoding and re-encoding, so it will not cause the decline in the quality of video. Meanwhile, the computation cost is low. The disadvantage is that the compression bit rate constraints the data volume embedded into watermark. 
This paper proposes a method that embeds watermark into the non coverage area of I frame of MPEG-4 streams. It is divided into several steps. First of all, the embedded digital watermarking should be concentrated in order to reduce its redundancy. Then find the areas that watermark can be embedded into on I frame. Watermark is embedded into this area of I frame and is directly embedded into the compressed bit rate. Because it is embedded into the non coverage area of I frame, watermark can be detected in each frame.

\section{Watermark Concentrating Algorithm}

The watermark that is embedded into image can be characters, images and so on[4]. The amount of information coding of character is minimum, but its stability is poor. It can become garbled easily after attack, so most of the time we use watermark in the form of picture. To simplify the discussion, this paper use binary image of $64 * 64$ size as an example.

Using binary image will bring about a large number of redundancy. As shown in Figure 1,

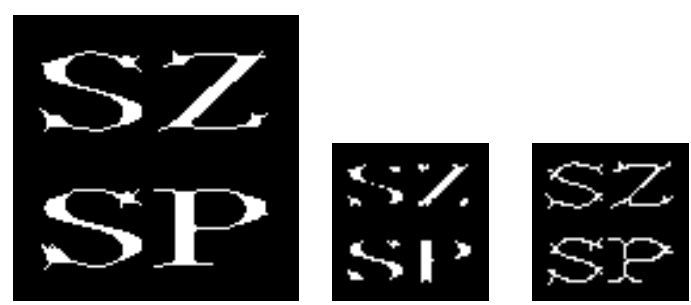

a. $64 * 64$ b. $32 * 32$ after skeleton scaling c. $32 * 32$ after simple scaling

Fig.1 Digit watermarking in different solutions

We can see that watermark (Figure 1.a) of $64 * 64$ size can carry the same amount of information as watermark (Figure 1.b) of $32 * 32$ size. But if we want to embed it into image, the latter has a quarter of the size of coded data as the former. If we can concentrate watermark information, we will reduce the modification on the original image. Then the similarity of watermark image will be improved.

If we simply reduce watermark image, we will lose parts of information of watermark. This will not realize the vindicability (Figure 1.c). For that, this paper proposes a new watermark information concentrating method-the skeleton concentrating method.

The basic idea of skeleton concentrating method is to extract regional skeleton of watermark containing information through thinning processing. Then using the improved method of reducing make watermark image keep the shape of skeleton as the watermark image reduces.

Thinning Processing. Thinning processing is often used in the preprocessing of character recognition which aims to search the skeleton of images and removes the redundant pixels in the image. So it will reduce the amount of information on the premise that we do not change the main features of image. Skeleton concentrating method requests us to remove the points of the outer edges of the object but keep the object not crack, that is, maintain the Euler number unchanged.

On the process of thinning processing, for one part, if the removed pixels are too few, the amount of information of image will not be reduced efficiently. For another part, if the removed pixels are too many, especially if some key points are removed, the main features of original image will be changed.

After this processing[5], we get the skeleton of watermark, as shown in Figure 2:

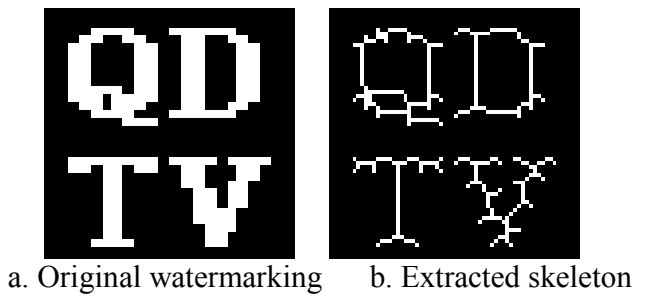

Fig.2 Skeleton extracting of watermarking

We can see from Figure 2 that the extracted skeleton is one pixel wide. When there are some special needs, we can keep the interior points. The width of the skeleton generated based on interior points retained thinning algorithm can be more than one pixel. In addition, in order to keep the special marks of watermark, we do not remove the redundant branch lines. 
3.2 Skeleton Scaling. In the field of image scaling, most of the time we research the image magnification. Accordingly we research image reduction little[6]. Gao Jian presented a weighted averaging method of image zooming-out that samples in the adjacent area. This method is based on gray-scale image. As for the common binary image and single pixel watermark skeleton image skeletonized, this paper presents a new method and designs the termination conditions of scaling:

i Read it into the skeletonized watermark image img;

ii According to the propoption set, generate new reduced image imgResult;

iii Reduced coefficient is $\mathrm{k}=\mathrm{SIZE}(\mathrm{img}) / \mathrm{SIZE}$ (imgResult);

For each point of imgResult:

$$
\begin{aligned}
& \text { imgResult }(i, j) \\
& =\left\{\begin{array}{l}
1 \sum_{m=1, n=1}^{k} i m g(k *(m-1)+m, k *(n-1)+n) \geq 1 \\
0 \sum_{m=1, n=1}^{k} i m g(k *(m-1)+m, k *(n-1)+n)=0
\end{array}\right.
\end{aligned}
$$

iv If the branch number of imgResult is as the same as img, then:

\{

img $<=$ imgResult;

turn to I;

\}

Or, end, return to img.

\section{Realizing of Digital Video Watermarking}

Selection of Watermark Embedding Area of I Frame. I frame is usually the first frame of every GOP (Grop of Picture). It is an independent frame with all the information. P frame refers to I frame. It is predicted from $P$ frame in front of it or from I frame. It compares the same information or data with $\mathrm{P}$ frame in front of it or I frame. It also considers the characteristics of motion to carry on interframe compression. P frame is based on the different points of the adjacent previous frame (I frame or P frame) to compress its data.

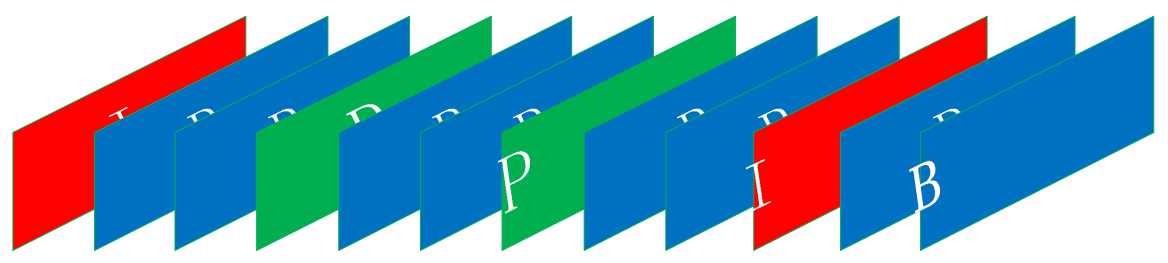

Fig.3 GOP components

In broadcasting system, when we do frame precision editing, because there is only a real I frame after a long time, we must calculate all the B frame and P frame in the range of GOP. Only when I frame comes can we carry out the editing of frame progress and the special effect processing. This is the LONG GOP material local high speed temporary decoding method that all non-compiled edition use. It is also the editing accuracy. In fact, in the radio and television equipment, the compression format that is truly used in large scale is MPEG2 I only format, that is, only MPEG2 of I frame which also calls MPEG2 422p@ML. Due to the abandoning of IBP long GOP structure, MPEG2 I frame format with $50 \mathrm{Mbps}$ bit rate in standard definition is usually considered to be broadcast quality. $120-180 \mathrm{Mbps}$ in high definition is considered to be broadcast editorial quality. $100 \mathrm{Mbps}$ is the acceptable broadcast quality. When broadcasting, the approach of radio and television systems is to save the transmission bandwidth and storage pressure. Before the data from production domain to broadcast domain is transmitted, MPEG2 I frame will convert to MPEG2 IBP short GOP or long GOP format. 
In the broadcast programs, especially the news program, the change of the background is not severe, so its area is relatively stable. If we find the unchanged area between two I frame and embed watermark into this area, any frame in this GOP can detect the embedded watermark in theory. Specific algorithm is as follows:

i Read I frame of current GOP, copy it to temp image variable ;

ii Read the next frame, if it is $\mathrm{P}$ frame, find its changing area and superimpose it to temp; if it is B frame, then turn to ii, else terminate the selection of current GOP embedding areas;

iii Return to temp image variable for the next watermark embedding ;

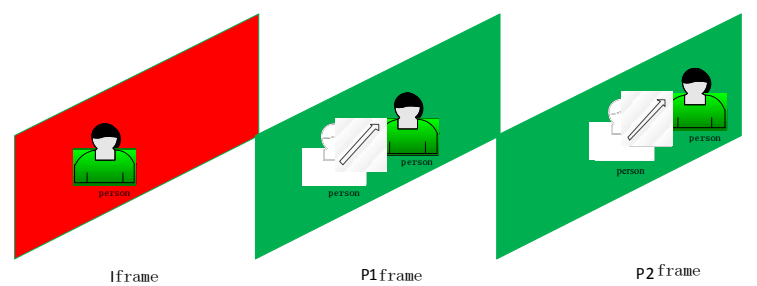

Fig.4 Sketch map of current GOP embedding area selection

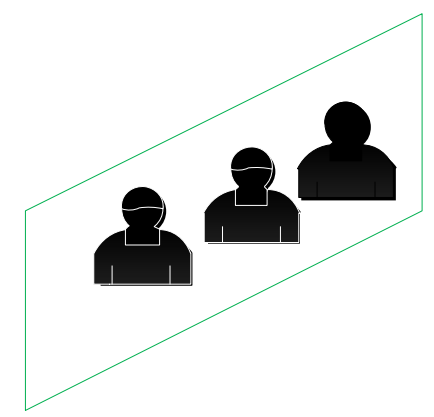

Fig.5 Get the embedded area of I frame of current GOP

Embedding Process of Watermark in I Frame. Because the GOP embedding areas obtained above are incoherent images, this paper chooses airspace domain watermark embedding method. Similar to the FAQ algorithm of literature [7], find the proper Harris feature points and choose the best embedding position in the current GOP available for embedding area acquired above. Under normal circumstances, we will extract multiple local areas. In this paper watermark will be repeatedly embedded into lots of areas to improve the robustness.

Harris corner detection algorithm extracts the feature points through detecting the position of mutation on local area of image. Harris corner detection algorithm can be matched to the characteristic points from I frame. We should remove the part dropping in the GOP changing area. As shown below:

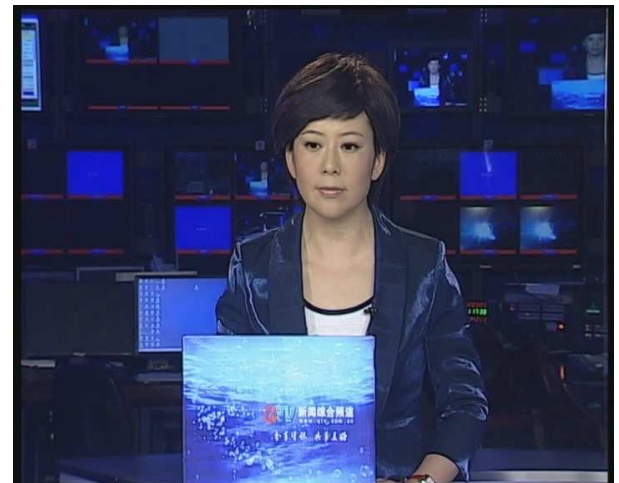

I Frame

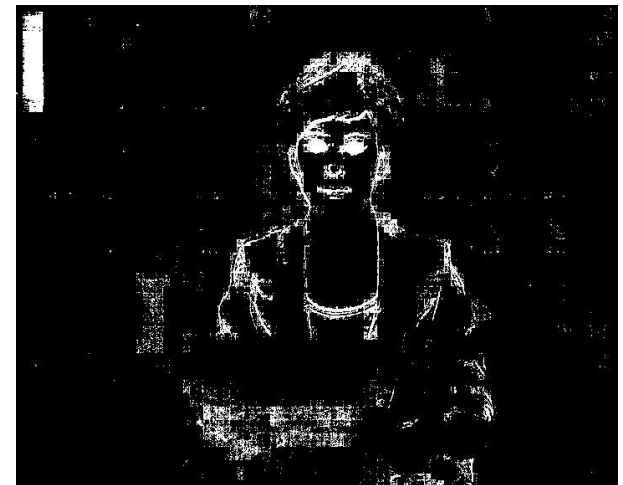

Changing area of GOP 


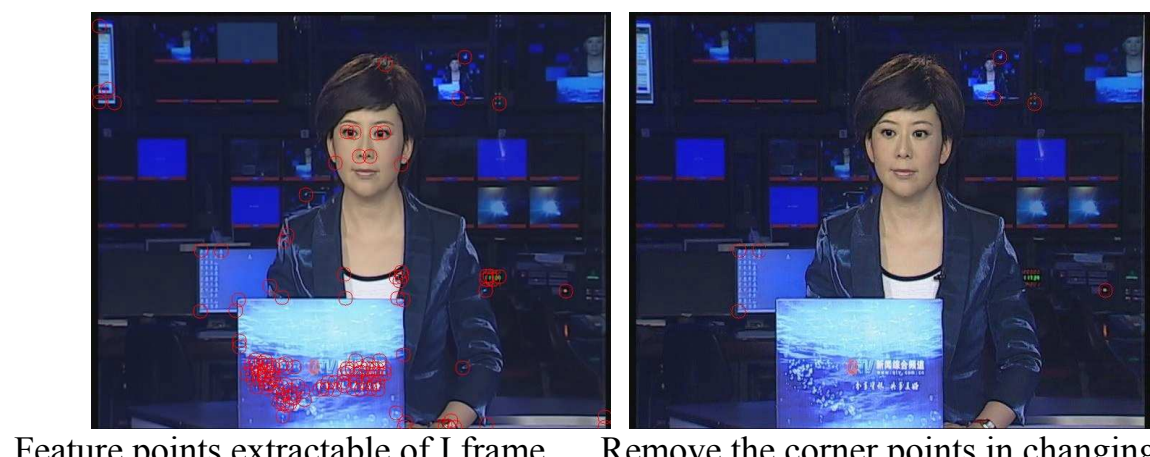

Feature points extractable of I frame Remove the corner points in changing area

Fig.6 Embedding position of watermark on I frame

The process of embedding and extracting watermark on I frame can refer to the FAQ method proposed by Li Lei-da[7]. After watermark is embedded into I frame, we get modified I frame. Replace the original I frame with the modified I frame of this GOP. Because P frame and B frame of this GOP are both acquired through decoding on this basis and watermark is embedded into uncovered area, any frame of this GOP can detect watermark in theory.

\section{Experimental Results}

This experiment uses VC and Opencv as experimental platform. According to the funding requirements, watermark is embedded into the $720 \times 576$ high definition television news programs. Watermark uses meaningful binary image of size $64 * 64$. Frame rate is 25 frame/s and one GOP is made up of one I frame and nine P frame.
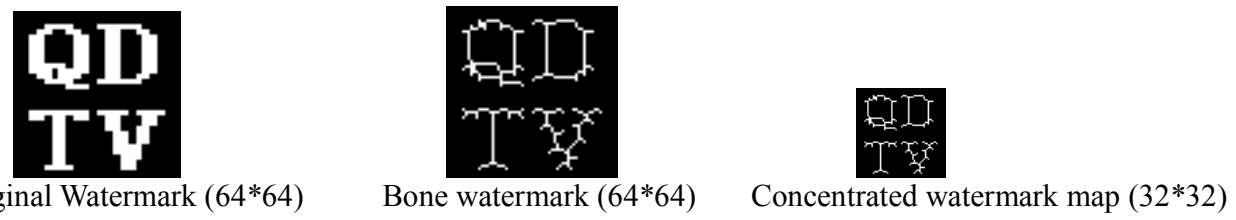

Fig.7 Watermark to be embedded

We use the methods above to embed and extract watermark in a number of television news programs, where $\mathrm{NC}=0.9508, \mathrm{PSNR}=35.449$.
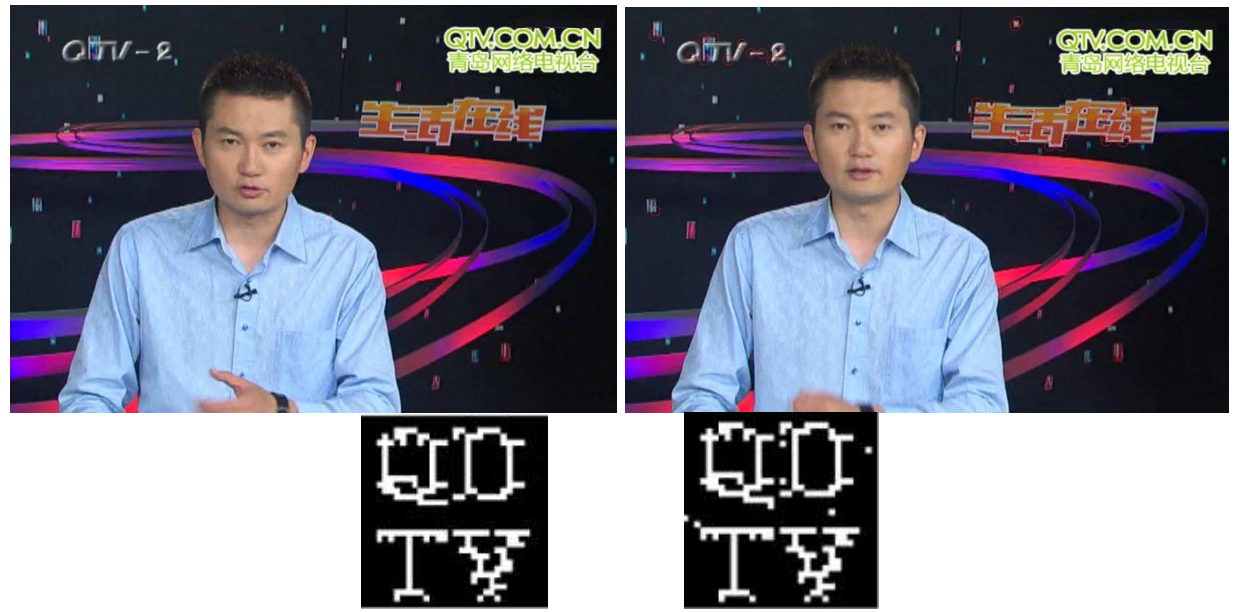

Fig.8 Watermark embedded and extracted after being concentrated

It can be seen from the above experiments that the essence of the video watermarking algorithm for TV program stream is to concentrate the embedded watermark image and greatly reduce the size of data you want to embed (The above experiment reduced to $1 / 4$ of the original), then embed the compressed watermark into the unchanged area of I frame of GOP. Watermark will be detected in the subsequent frames. 


\section{Conclusion}

This paper proposed a new digital video watermarking algorithm for TV Program stream. It concentrates the watermark image and reduces the size of embedded data. The compressed watermark signal is embedded into the unchanged area of I frame. The main advantages of this method have:

$1>$ Concentrated watermark image reduces the size of embedded data, reducing the damage to the original image and improving the invisibility;

$2>$ Watermark is embedded into the unchanged area of I frame of the original video. We just need computing resource as watermark is embedded. The TV program does not change while broadcasting. It will not increase the amount of computation;

$3>$ Because the area that watermark is embedded into is the unchanged area of I frame, the subsequent frames all decode by means of this I frame. Watermark can be detected in subsequent frames.

Experiments show, the algorithm can not only ensure good invisibility of the embedded watermark, but also well balance the requirements of the invisibility and the computational cost.

However, this algorithm requires that there is an unchanged area with certain size, or we can not find the proper position for watermark to be embedded into. In the follow-up study we will work to change this problem.

\section{Acknowledgment}

Science and Technology Support Program of Qingdao Public Sphere funded.

\section{References}

[1] Deguillaume F, Csurka G, and Ruanaidh J O,et al.. Robust 3D DFT video watermarking. Proceedings of SPIE Security and Watermarking of Multimedia Contents, San Jose, USA, 1999, 3657: 113-124.

[2] Zhang Li-he, Wu Hong-tao, Hu Chang-li. A Video Watermarking Algorithm Based on 3D Gabor transform[J]. Journal of software, 2004, 15(8): 1252-1258.

[3] Tian Yu-min, Qu Yun-hui, Wan Bo. A Digital Video Watermarking Algorithm Based on MPEG-4 Streams [J]. Journal of Shenzhen University, 2008, 25(2):173-177

[4] Jin Yuan-zhi, Zhou Hui-juan, Zhang Yan-ke, etc. Resisting Geometric Attacks Image Watermarking Algorithm Based on Object[J]. Application Research of Computers, 2009(011): 4312-4314.

[5] Liu Li-na,Wu Xi-sheng. Improved Algorithm of Enhancing Handwritten Digit Thinning Effect [J]. Computer Engeineering and Applications, 2010, 3.

[6] Gao Jian, Mao Shi-qun, Zhou Yu-zheng, etc. A Weighted Averaging Algorithm of Image Zooming-out Based on Image Sub-block [J]. Journal of Image and Graphics, 2006, 11(010): $1460-1463$.

[7] Li Lei-da, Guo Bao-long, Wu Xiao-yue. A New Spatial Domain Image Watermarking Scheme Resisting Geometric Attacks [J], ACTA AUTOMATICA SINICA, 2008,10:1235-1242 